\title{
Morphopathological and immunohistochemical features of a pure mucinous breast carcinoma - Case report
}

\author{
${ }^{1}$ Associated member of Academy of Romanian Scientists \\ ${ }^{2}$ Department of Pathology, Faculty of Medicine, "Ovidius" University, Constanta \\ ${ }^{3}$ Clinical Service of Pathology, Emergency County Hospital "Sf. Apostol Andrei” Constanta
}

\begin{abstract}
Pure mucinous carcinoma is a rare special type of breast carcinoma with a $2 \%$ incidence and it is usualy asociated with a good prognosis. It must distingished from the mixed subtype of mucinos breast carcinoma, which has an invasive non-mucinous component in more than $10 \%$ of the tumor and change the favourable outcome of the first subtype. In this report we present a case of a premenopausal woman with a lump in right breast wich histopathologically proved to be a pure mucinous carcinoma associated with high grade ductal carcinoma in situ. Immunohistochemical and ancillary studies demonstrate a great heterogeneity of the neoplastic cells, with different molecular profile for each component of the tumor. The presence of ductal carcinoma in situ with a different imunophenotype from pure mucinous carcinoma rise the ipothesis of a different tumor cell biology which may change clincal evolution.
\end{abstract}

Keywords: Pure mucinous breast carcinoma, ductal carcinoma in situ, immunohistochemistry, CISH

\section{Gabriela Izabela Baltatescu}

Department of Clinical Service of Pathology, Emergency County Hospital,

145 Tomis Avenue, 900591 Constanta, Romania

email : gabrielabaltatescu@yahoo.com

phone: +40241503289

\section{Introduction}

Despite numerous prevention and screening programs for early detection of breast carcinoma (BC), it still ranks second worldwide incidence and the leading cause of mortality among women, especially in the developing countries $[1,2]$. It is responsible for 1.7 milion of all new cases of $\mathrm{BC}$ and for more than a half of milion of deaths according to the latest data [2].

Mucinous carcinoma is a rare special type of $\mathrm{BC}$ with $2 \%$ from all breast cancer and is defined as pools of extracelular mucin in wich cluster of malignant cells are floating [3]. When more than $90 \%$ of the tumor is represented by a mucinous morphology, than it is considered as pure subtype of mucinous breast carcinoma, wich is associated with an excellent prognosis as demonstrated by previous studies $[4,5]$. The second subtype of mucinous $\mathrm{BC}$ is the mixed-type and it is considered as a diagnose when the non-mucinous component is present in more than $10 \%$ of the tumor [6]. The most frequent type of the non-mucinous component is invasive ductal carcinoma - no special type (IDC-NST) [7]. The mucinous carcinoma prognosis worsens when it 
is associated with a ductal invasive carcinoma, being similar to that of the later component [8].

In this report we present a case of a premenopausal woman with a lump in right breast wich histopathologically proved to be a pure mucinous carcinoma associated with solid and comedo-type ductal carcinoma in situ (DCIS). Immunohistochemical and ancillary studies demonstrate a great heterogeneity of the tumor cells which sugest a different molecular pathways of progression.

\section{Materials and mehods}

Case report. A 47 years old woman was admitted in the surgical department of The Hospital County Constanta for a tumor mass in her upper-outer quadrant of the right breast. The lesion was detected by the patient 7 months ago and her clinical history did not notice anything significant. Mammography confirmed the presence of a well-circumscribed lobulated nodule with radiologic features highly sugestive for malignancy. Computer tomography or clinical examination revealed no other abnormalities. The sectorectomy was performed and frosen sections proved the clinical suspicions of invasive carcinoma.

Methods. Tumor samples were selected and $4 \mu \mathrm{m}$ thick sections of formalin fixed, paraffinembedded tissue block of the tumor were stained with hematoxylin and eosin. Microscopic examination establieshed the histopathological type of BC using the criteria recommended by the World Health Organization [3] and grade according to the ScarffBloom-Richardson scale modified by Elston and Ellis [9]. After epitope retrieval, immunostain was performed on the best representative slides using a pannel of four antibodies from DakoCytomationDenmark (Tabel 1). 3,3'diaminobenzidine (DAB) was used as chromogen, resulting a brown stain of the antigen and the final stage was Mayer's haematoxylin as a counterstain. The semiquantitative scoring method was applied for immunohistchemical expression of hormonal estrogen and progesteron receptors and the cut-off value for positive result was $1 \%$ nuclear stain [10]. Recomandations of Dowsett et al. were used for evaluation of Ki67 immunostain, where the cut-off value for low or high expression was $14 \%$ nuclei stained [11]. Newest ASCO/CAP guidelines were applied for assesment of HER2 status (over-expression or absence of the HER2/ neu protein)[12]. For those cases with an equivocal result at immunohistochemistry, molecular study was performed in order to establised the cerbB2 gene amplification in tumor cells. Chromogenic in situ hybridisation (CISH) was the method used for this purpose with ZytoDot SPEC HER2 Probe Kit from ZytoVision and the results were evaluated according to manufacturer's criteria.

Table I The pannel of antibody used for immunohistochemical examination (source, clone, dilution)

\begin{tabular}{|c|c|c|c|}
\hline Antibody ${ }^{*}$ & Clona & Dilution & Antigen retrieval \\
\hline ER & $\begin{array}{l}\text { Rabbit monoclonal } \\
\text { antibody1D5 }\end{array}$ & $\begin{array}{l}\text { Ready to } \\
\text { use }\end{array}$ & $\begin{array}{c}\text { EnVision TM FLEX Target Retrievel } \\
\text { Solution, high PH }\end{array}$ \\
\hline PR & $\begin{array}{r}\text { Mouse monoclonal } \\
\text { antibody PgR } 636\end{array}$ & $\begin{array}{l}\text { Ready to } \\
\text { use }\end{array}$ & $\begin{array}{c}\text { EnVision TM FLEX Target Retrievel } \\
\text { Solution, high PH }\end{array}$ \\
\hline HER2neu & $\begin{array}{c}\text { Rabbit } \\
\text { Imunoglobulin } \\
\text { HercepTest }\end{array}$ & $\begin{array}{l}\text { Ready to } \\
\text { use }\end{array}$ & 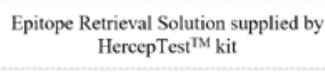 \\
\hline Ki-67 & $\begin{array}{l}\text { Mouse monoclonal } \\
\text { antibody MIB-1 }\end{array}$ & $\begin{array}{l}\text { Ready to } \\
\text { use }\end{array}$ & $\begin{array}{c}\text { EnVision TM FLEX Target Retrievel } \\
\text { Solution, high PH }\end{array}$ \\
\hline
\end{tabular}

\section{Results}

Macroscopic evaluation of the surgical specimen revealed a solid mass measuring $2.5 \times 1.8$ $\mathrm{cm}$, well-defined, firm and white-tan color with translucid areas (Figure1).

Morphopathological examination observed clusters of malignant cells floating in lakes of mucus, with low nuclear grade (Figure 2A) characteristic for subtype A (hypocellular) of pure mucinous BC. An invasive component of moderate differentiated IDC -NST was identified, but because it didn't exceed 10\% of the tumor volume, then pure mucinous carcinoma was the correct diagnose (Figure 2B). It was also observed an intratumoral component represented by 
solid and comedo-type DCIS with high nuclear grade (Figure 2C).

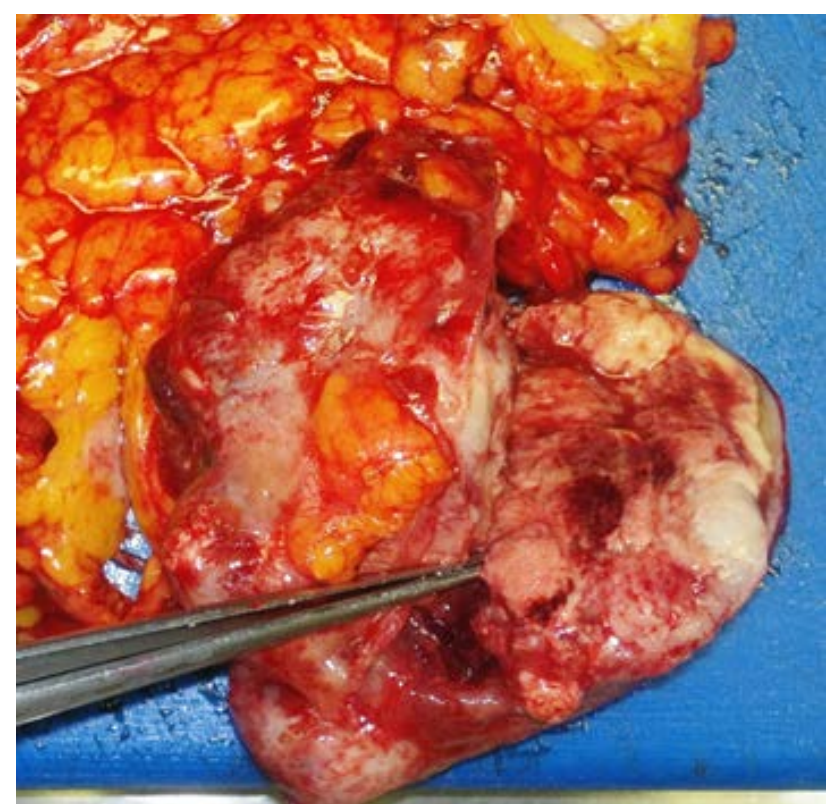

Figure 1 Macroscopic aspect of pure mucinous carcinoma: a well-circumbscribed solid tumor with a lobulated contour, white-tan colour and translucid areas

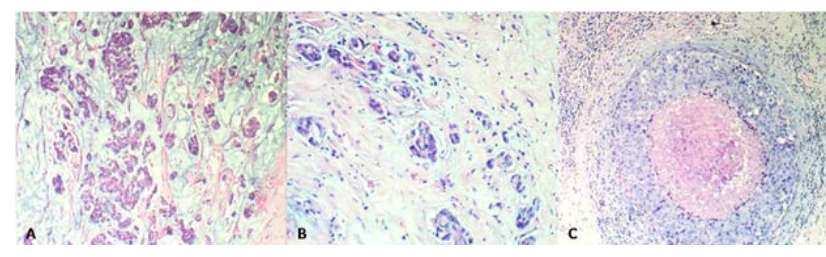

Figure 2 Microscopic features of the tumor: A) mucinous $B C$ with pools of mucin in which clusters of tumor cells are floating (HE, 4x); B) IDC-NST component (HE, 4x); C) comedo-type DCIS (HE, 10x)

Immunohistochemical examination showed different patterns of expression for each biomarkers and also for the different component of the tumor. It was noticed a high level of expression for hormonal receptors (ER and PR) in the mucinous compartment in contrast with moderate expression for IDC-NST and none for DCIS (Figure 3.A,B,C).

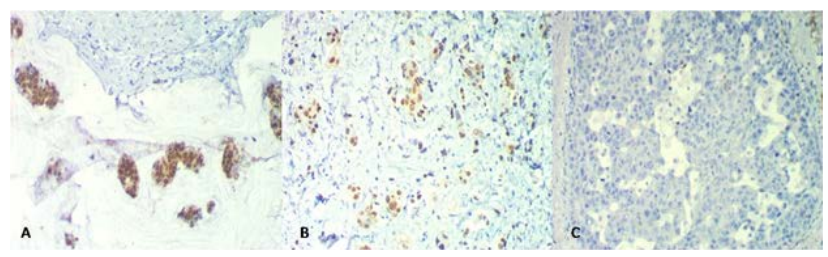

Figure 3 ER immunostain: A) intens nuclear stain in the tumor cells of the mucinous BC (IHC, 10x); B) moderate nuclear imunostain of IDC-NST (IHC, 10x);C) absence of ER expresion in the tumor cells of DCIS (IHC, 10x)

It was also obtained different IHC scores when overexpression of HER2neu oncoprotein was evaluated by immunohistochemistry: IHC 0 - negative for mucinous BC (incomplete and faint membrane stain in less than $\leq 10 \%$ of malignant cells); IHC 2- equivocal in IDC-NST (incomplete and weak to moderate membrane stain within $>10 \%$ of neoplastic cell); IHC +3 - positive for DCIS (intense and complete membrane stain in more than $10 \%$ of malignant cell) (Figure 4.A,B,C). The rate of nuclear proliferation proved to be low because the Ki67 index is less than $14 \%$ within all tumor cells.

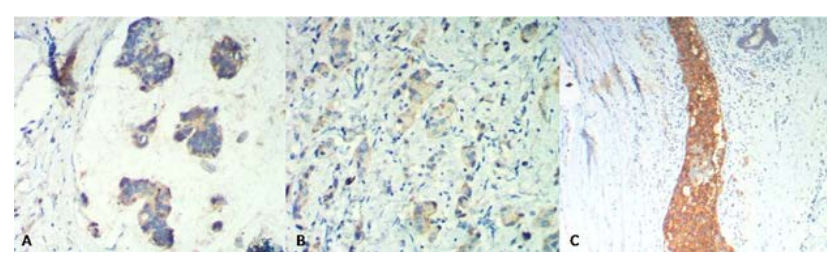

Figure 4 Immunohistochemical HER2neu oncoprotein status: A) IHC score 0 -negative in mucinous BC (IHC, $10 x)$; B) IHC score 2 - equivocal in the DCI-NST (IHC,

10x); C) IHC score 3 - positive in DCIS (IHC, 4x)

Molecular study was further performed to evaluate HER2neu gene amplification. The result for CISH exam varied depending on tumor components: mucinous BC and IDC-NST with no amplification, both had shown a normal nuclei each with two HER2 signals (Figure 5 A,B); DCIS with a high level of amplification, characterised by a mixture of numerous small clusters and rare big clusters, with more than 7 signals HER2 present per nucleus (Figure 5C). 


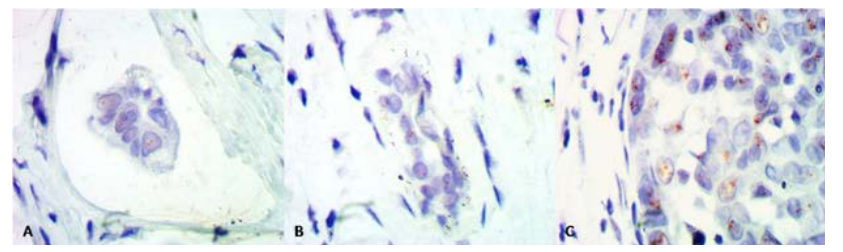

Figure 5 HER2neu gene status by CISH: no amplification for mucinous BC component (A) and for IDC-NST component (B) (20x); high level of HER2neu gene amplification in DCIS component (C) (20x)

\section{Discussion}

Mucinous breast carcinoma with its synonyms (colloid BC, mucoid carcinoma; mucinous adenocarcinoma) is one of the rarest special subtypes of $\mathrm{BC}$ with an incidence range between $1 \%$ to $2 \%$ $[3,8]$. Most cases are diagnosed after the fifth decade of life, frequently of white ethniticity [13]. The mean age 68.3 yo obtained by a large retrospectiv study proved that it is statistical significantly higher than that observed in cases with IDC-NST (61 yo) [3, 13]. Even if our patient is younger than the mean age observed in large data base studies, it still fall between the values recorded of 21 to 94 yo [14]. As in the present case, most of the tumors are located in the upper outer quadrant (44\%) and also the tumor diameter is close to the $2.2 \mathrm{~cm}$ mean value observed in different study, but larger tumors up to $25 \mathrm{~cm}$ were also described $[13,15]$. Morphopathological features of the tumor consist with subtype A of pure mucinous $\mathrm{BC}$ (hypocellular type), the most frequent type but there are also described forms with hipercellularity (subtype B) or with intermediate features (subtype AB), with no impact on prognosis [8]. The only differences between subtype A and B are the age of the patient at the time of diagnose (older patient for subtype B) and the presence of neuroendocrine differentiation in subtype B [16]. The mucinos component of our case is well differentiated which is consisted with previous reports, where well differentiated tumors had been found in up to $65 \%$ of subtype A mucinous BC [17]. The presence of an IDC-NST in less then $10 \%$ of the tumor have imposed exclusion of a mixt mucinous $\mathrm{BC}$ diagnose [3]. This has a profound impact on prognosis, because the survival rate for pure mucinous $\mathrm{BC}$ is better than the mixt mucinous $\mathrm{BC}$ wich has a similar prognosis to that of IDC-NST [5]. In a large study with 1221 pacients with mucinous $\mathrm{BC}$, the overall survival was $80 \%$ versus $77 \%$ for IDC-NST [18].

DCIS is frequently found in the pure mucinous $\mathrm{BC}$, usually at the periphery of the tumor and it has been discribed all morphological subtypes [19]. In the study of Gadre SA et al, solid DCIS was identified in almost half of cases, comedo-type DCIS up to $30 \%$ cases of mucinous BC, most of the well and moderate differentiated and only $11 \%$ of cases with high nuclear grade as in our report [20].

Numerous immunohistochemical studies on mucinous $\mathrm{BC}$ show that almost all cases are positive for hormonal receptors, especially for ER. The ER expression varied from $73.4 \%$ [4] to $100 \%$ [21] positivity, which are higher compared with those for PR: $65.4 \%$ [4] to $85.7 \%$ [22]. It was also proved positive immunostain for androgen receptor (AR) but with a high range of values: $1.7 \%$ [23] $-21.7 \%$ [24]. This hormonal profile argue the good prognosis of this specific subtype of BC. In present case, it was also obtained a positive results for hormonal receptor both in the mucinous and in the IDC-NST component consisted with literature reports. The absence of ER and PR expression in the in situ component wasn't surprising because it had been demonstrated that highgrade DCIS, especialy comedo-type has a negative ER and PR imunostain [25, 26, 27]. Both biomarkers have a predictive role since patients with a positive imunostain receive treatment with trastuzumab [28]. More than that, it has been proved that high-grade DCIS with absence of ER expression is associated with a high risk of recurrence [29].

The proliferation rate of tumor cells was assesed using Ki67 imunostain and it was observed a low Ki67 index for all components of the tumor. These values are in concordance with previous reports which consistently showed low rate of Ki67 expression for numerous cases of pure mucinous BC, up to $91.4 \%$ in one study [22]. On the other hand, in 
IBC and DCIS proliferation rate evaluated by Ki67 imunostain recorded different values from low to high rates and it is usualy correlated with tumor grade [30]. Recently, a high KI67 index proved to be an independed prognostic factor, those patients having a high risk for recurrence as IBC [31]

HER2neu oncoprotein is one of the four memberes of HER family and plays and important role in cellular growth [32]. If for IBC, the negative impact on prognosis of HER2neu overexpresion is well establised, its role is still debate in DCIS [33]. The study of Borgquist S. et al. proved that its positivity is associated with lower risk of recurrent invasive BC [34]. Recently, it was demonstrated that HER2neu amplification implies a high risk for DCIS recurrence [35]. Also it has been demonstrated that HER2neu amplification is aproximatively three time higher in DCIS than IBC-NST [36]. In adition, HER2neu overexpresion in high-grade DCIS is frequently associated with extensive comedo-type necrosis, a high rate of nuclear proliferation and with a negative hormonal status [36]. In contrast, mucinous $\mathrm{BC}$ is usualy negative to HER $2 \mathrm{neu}$, only $5 \%$ of cases being reported as positive [18]. In present case, we observed a heterogenity of HER 2 neu immunostain from negative, in mucinous component, to an equivoc result for the small IBC-NST component and positive in DCIS component. CISH examination demonstrated the absence of HER2neu amplification in IDC-NST and in mucinous componet, in contrast with high level of amplification for tumor cell of in situ component.

So, it was noticed a different molecular profile for different areas of the tumor: $\mathrm{ER}+/ \mathrm{PR}+/ \mathrm{HER} 2$ neu - for mucinous BC and IBC-NST versus ER-/PR- / HER2neu + for DCIS. These results rise the ipothesis of a different tumor cell biology for this type of breast lesions, which may have a unfavourable impact on prognosis.

\section{Conclusions}

Pure mucinous breast carcinoma in a premenopausal women is a very rare special subtype of $\mathrm{BC}$ and usualy are assoicated with a favourable prognosis. Even if it is associated with an excelent prognosis, the presence of DCIS with a different imunophenotype may change clincal evolution of this tumor. Because its consecutively high risk for an in situ carcinoma recurrence, the patient mast be consistently evaluated.

\section{References}

1. Ferlay, J., Soerjomataram, I., Ervik, M., et al. (2013) GLOBOCAN 2012 v1.0, Cancer Incidence and Mortality Worldwide: IARC Cancer Base No. 11. Lyon, France: International Agency for Research on Cancer. Retrieved March 3, 2016 from: http://globocan.iarc.fr

2. Global Cancer Facts \& Figures 3rd Edition. Retrieved March 5, 2016 from http://www.canc er.org/acs/groups/content/@research/

3. Bussolati, G. \& Sapino, A. (2012). Mucinous carcinoma and carcinoma with signet-ringcell differentiation. In Lakhani S., Ellis I., Schnitt S., et al. (Eds.) WHO Classification of Tumours of the Breast $4^{\text {th }}$ ed. (pp60-61). Lyon: IARC Press.

4. Cao, A.Y., He, M., Liu, Z.B., Di, G.H., Wu, J., Lu, J.S., Liu, G.Y., Shen, Z.Z. \& Shao, Z.M. (2012). Outcome of pure mucinous breast carcinoma compared to infiltrating ductal carcinoma: a population-based study from China. Ann Surg Oncol. Sep 19(9):3019-27. doi: 10.1245/s10434-012-2322-6. 19:30193027.

5. Bae, S.Y., Choi, M.Y., Cho, D.H., et al. 
(2011). Mucinous carcinoma of the breast in comparison with invasive ductal carcinoma: clinicopathologic characteristics and prognosis. J Breast Cancer.14:308-13. doi: 10.4048/jbc.2011.14.4.308.

6. Sahoo, S. \& Shin, S.J. (2012). Special types of breast carcinoma: tubular carcinoma, mucinous carcinoma, cribriform carcinoma, invasive micropapillary carcinoma and medullary carcinoma. In Dabbs DJ. Breast pathology. (pp 536-572). Elsevie Saunder.

7. Kashiwagi, S., Onoda, N., Asano, Y., Kawajiri, H., Takashima, T., Ohsawa, M., et al. (2013). Clinical significance of the subclassification of 71 cases mucinous breast carcinoma. Springer plus. 2:481-86.

8. Corben, A.D. \& Brogi, E. (2014) Mucinous carcinoma. In Hoda, S.A., Brogi, E., Koerner, F.C. \& Rosen, PP. (Eds.) Rosen's breast pathology- fourth edition. (pp 611644). Philadelphia: Lippincott Williams \& Wilkins.

9. Elston, C.W.\& Ellis, I.O. (1991). Pathological prognostic factors in breast cancer. The value of histological grade in breast cancer: experience from a large study with long-term follow-up. Histopathology. 19 (5): 403-10.

10. Hammond, M.E., Hayes, D.F., Allred, D.C., Dowsett, M., Hagerty, KL., Badve, S., et al. (2010) American Society of Clinical Oncology/College of American Pathologists Guideline Recommendations for Immunohistochemical Testing of Estrogen and Progesterone Receptors in Breast Cancer. Arch Pathol Lab Med. 134(7):e48-e72.

11. Dowsett, M., Nielsen, T.O., A'Hern, R., Bartlett, J., Coombes, C., Cuzick, J., Ellis, M. et al. (2011). Assessment of Ki67 in Breast Cancer: Recommendations from the International Ki67 in Breast Cancer Working Group. J Natl Cancer Inst. 103(22):16561664.

12. Wolff, A.C., Hammond, M.E., Hicks, D.G.,
Dowsett, M., McShane, L.M., Allison, K.H. et al. (2013). Recommendations for human epidermal growth factor receptor 2 testing in breast cancer: American Society of Clinical Oncology/College of American Pathologists clinical practice guideline update. $J$ Clin Oncol 31(31):3997-4013. doi: 10.1200/ JCO.2013.50.9984.

13. Di Saverio, S., Gutierrez, J. \& Avisar, E. (2008). A retrospective review with long term follow up of 11,400 cases of pure mucinous breast carcinoma. Breast Cancer Res Treat. 111:541-547.

14. Sahoo, S. \& Shin, S.J. (2012) Special types of breast carcinoma: tubular carcinoma, mucinous carcinoma, cribriform carcinoma, invasive micropapollary carcinoma and medullary carcinoma. In Dabbs DJ.(Eds) Breast pathology. (pp 536- 572). Elsevier Saunders

15. Ishikawa, T., Hamaguchi, Y., Ichikawa, Y. et al. (2002). Locally advanced mucinous carcinoma of the breast with sudden growth acceleration: a case report. Jpn J Clin Onc. 32:64-67.

16. Scopsi, L., Andreola, S., Pilotti, S., et al (1994). Mucinous carcinoma of the breast. A clinicopathologic, histochemical, and immunocytochemical study with special reference to neuroendocrine differentiation. Am J Surg Pathol.18:702-711.

17. Ranade, A., Batra, R., Sandhu, G., et al (2010). Clinicopathological evaluation of 100 cases of mucinous carcinoma of breast with emphasis on axillary staging and special reference to a micro papillary pattern. J Clin Pathol. 63:1043-1047.

18. Diab, S.G., Clark, G.M., Osborne, C.K., et al (1999). Tumor characteristics and clinical outcome of tubular and mucinous breast carcinomas. J Clin Oncol. 17:1442-1448

19. Fentiman, I.S., Millis, R.R., Smith, P., et al. (1997) Mucoid breast carcinomas: histology 
and prognosis. $\mathrm{Br} T$ Cancer. 75:1061-1065.

20. Gadre, S.A., Perkins, G.H., Sabin, A.A., et al. (2008) Neovascularization in mucinous ductal carcinoma in situ suggests an alternative pathway for invasion. Histopathology. 53:545-553.

21. Slodkowska, E., Corben, A.D., Catalano, J.P., et al (2012). Pure mucinous carcinoma in women 40 years old or younger: clinicopathological and follow-up study. USCAP Meeting Vancouver Canada, March 2012.

22. Lacroix-Triki, M., Suarez, P.H., MacKay, A., et al. (2010). Mucinous carcinoma of the breast is genomically distinct from invasive ductal carcinomas of no special type. $J$ Pathol. 222:282-298.

23. Park, S., Koo, J., Park, H.S., et al. (2010) Expression of androgen receptors in primary breast cancer. Ann Oncol. 21:488-492.

24. Cho, L.C. \& Hsu, Y.H. (2008) Expression of androgen, estrogen and progesterone receptors in mucinous carcinoma of the breast. Kaohsiung I Med Sci. 24:227-232

25. Rauch, G.M., Kuerer, H.M., Scoggins, M.E., Fox, P.S., et al (2013). Clinicopathologic, mammographic, and sonographic featuresin 1,187 patients with pure ductal carcinoma in situ of the breast byestrogen receptor status. Breast Cancer Res Treat 139:639-647. doi:10.1007/s10549-013-2598-7.

26. Lari, S.A. \& Kuerer, H.M. (2011) Biological markers in DCIS and risk of breast recurrence: a systematic review. $J$ Cancer. 2:232-261. doi:10.7150/jca.2.232.

27. Walker, R.A., Hanby, A., Pinder, S.E., et al.(2012) Current issues in diagnostic breast pathology. J Clin Pathol. 65:771-785

28. Allred, D.C., Bryant, J., Land, S., et al. (2002) Estrogen receptor expression as a predictive marker of the effectiveness of tamoxifen in the treatment of DCIS: findings from NSABP protocol B-24. Breast Cancer Res. Treat. 76 (Suppl. 1), (abstract 30).
29. Roka, S., Rodas, M., Taucher, S., et al. (2004) High nuclear grade and negative estrogen receptor are significant risk factors for recurrence in DCIS. Eur J Surg Oncol. 30:243-247.

30. Wiechmann, L. \& Kuerer, H.M. (2008). The molecular journey from ductal carcinoma in situ to invasive breast cancer. Cancer. 112:2130-2142. doi:10.1002/cncr.23430.

31. Williams, K.E., Barnes, N.L.P., Cramer, A., Johnson, R., et al (2015) Molecular phenotypes of DCIS predict overall and invasive recurrence. Ann Oncol. 26(5):10191025

32. Lohrisch, C. \& Piccart, M. (2001) An overview of HER2. Semin Oncol. 28:3-31.

33. Latta, E.K., Tjan, S., Parkes, R.K. \& O'Malley, F.P. (2002) The role of HER2/ neu overexpression/amplification in the progression of ductal carcinoma in situ to invasive carcinoma of the breast. Mod Pathol. 15:1318-25.

34. Borgquist, S., Zhou, W., Jirström, K., Amini, R.M., Sollie, T., Sørlie, T., et al (2015). The prognostic role of HER2 expression in ductal breast carcinoma in situ (DCIS); a populationbased cohort study. BMC Cancer. Jun 11;15:468. doi: 10.1186/s12885-015-1479-3.

35. Hoque A, Sneige N, Sahin AA. (2002). Her-2/ neu gene amplification in ductal carcinoma in situ of the breast. Cancer Epidemiol Biomarkers Prev. 11:587-590.

36. Bockstal MV., Lambein K., Denys H., Braems G. et al. (2014) Histopathological characterization of ductal carcinoma in situ (DCIS) of the breast according to HER2 amplification status and molecular subtype. Virchows Arch. 465:275289; doi 10.1007/s00428-014-1609-3 